\title{
SENSITIVITY OF PARTICLE EXTINCTION AND BACKSCATTERING CALCULATION FROM MIE-RAMAN LIDAR MEASUREMENTS TO THE CHOICE OF ÅNGSTRÖM EXPONENT
}

\author{
Anastasia Suvorina $^{1}$, Igor Veselovskii ${ }^{1}$, David N. Whiteman ${ }^{2}$, Michael Korenskiy $^{1}$ \\ ${ }^{1}$ Physics Instrumentation Center, Troitsk, Moscow Region, 142190, Russia, E-mail: \\ E-mail: igorv@pic.troitsk.ru \\ ${ }^{2}$ Mesoscale Atmospheric Processes Laboratory, NASA Goddard Space Flight Center, Greenbelt, MD 20771, USA, \\ E-mail: david.n.whiteman@nasa.gov
}

\begin{abstract}
Vibrational Raman scattering from nitrogen is commonly used in Mie-Raman lidars for evaluation of particle backscattering $(\beta)$ and extinction $(\alpha)$ coefficients. However, vibrational scattering is characterized by significant frequency shift of the Raman component, so for the calculation of $\alpha$ and $\beta$ the assumption about the extinction Ångström exponent is needed. Simulation results presented in this study demonstrate that ambiguity in the choice of this exponent can be the significant source of uncertainty in the calculation of backscattering coefficients when optically thick aerosol layers are considered. Examples of lidar measurements and optical data calculated for different values of Ångström exponent are given.
\end{abstract}

\section{INTRODUCTION}

The particle backscattering $(\beta)$ and extinction $(\alpha)$ coefficients measured at multiple wavelengths with a Raman lidar can be inverted to particle microphysics. The system most often used for such measurements, due to its relative simplicity, is a Raman lidar based on a tripled Nd:YAG laser, which provides three backscattering ( $355 \mathrm{~nm}, 532 \mathrm{~nm}, 1064 \mathrm{~nm})$ and two extinction $(355 \mathrm{~nm}, 532 \mathrm{~nm})$ coefficients. To get this so called $3 \beta+2 \alpha$ data set the elastic backscatters are measured simultaneously with nitrogen vibrational Raman signal at $387 \mathrm{~nm}$ and $608 \mathrm{~nm}$. However the vibrational Raman scattering is characterized by significant wavelength shift of the nitrogen Raman component with respect to the laser line. As a result, information about the spectral dependence of aerosol extinction is needed when $\alpha$ and $\beta$ are computed, which can be an additional source of uncertainty. In our presentations we report the results of numerical simulations and analysis of experimental measurements aimed to estimate corresponding uncertainties.

\section{NUMERICAL SIMULATIONS}

The possibility of independent calculation of aerosol backscattering and extinction coefficients from simultaneously measured elastic and Raman backscatters was first demonstrated by Ansmann et al. [1]. Following their approach the backscattering and extinction coefficients at height $\mathrm{z}$ can be calculated as

$$
\begin{aligned}
& \alpha_{L}^{a}(z)=\frac{\frac{d}{d z} \ln \left(\frac{N_{R}(z)}{P_{R}(z) \cdot z^{2}}\right)-\alpha_{L}^{m}(z)-\alpha_{R}^{m}(z)}{1+\left(\frac{\lambda_{R}}{\lambda_{L}}\right)^{A}} \\
& \beta_{L}^{a}(z)=-\beta_{L}^{m}(z)+\beta_{L}^{m}\left(z_{0}\right) \frac{P_{R}\left(z_{0}\right) P_{L}(z) N_{R}(z)}{P_{L}\left(z_{0}\right) P_{R}(z) N_{R}\left(z_{0}\right)} \times \\
& \times \exp \left\{\int_{z_{0}}^{z}\left[\alpha_{L}\left(1-\left(\frac{\lambda_{L}}{\lambda_{R}}\right)^{A}\right)+\left(\alpha_{L}^{m}-\alpha_{R}^{m}\right)\right] d z^{\prime}\right\}
\end{aligned}
$$

Where $P_{L, R}(z)$ is the power of elastic and Raman backscatters, $\lambda_{\mathrm{L}, \mathrm{R}}$ are corresponding wavelengths and $\mathrm{N}_{\mathrm{R}}(\mathrm{z})$ is the density of Raman scatters. The reference height $\mathrm{z}_{0}$ is usually chosen in the free troposphere where $\beta_{L}^{a}\left(z_{0}\right)<<\beta_{L}^{m}\left(z_{0}\right)$. Indices " $\mathrm{a}$ " and " $\mathrm{m}$ " here correspond to aerosol and molecular components of backscattering and extinction coefficients.

To account for spectral dependence of $\alpha^{a}$ the extinction Ångström exponent (EAE) $A$ is used:

$$
\frac{\alpha^{a}\left(\lambda_{L}\right)}{\alpha^{a}\left(\lambda_{R}\right)}=\left(\frac{\lambda_{R}}{\lambda_{L}}\right)^{A}
$$

The value of EAE is unknown, besides it can be height and spectrally dependent, however in computations usually the fixed value of $A$ is assumed. Thus the ambiguity in choice of Ångström exponent 
is an additional source of uncertainties in $\beta^{a}$ and $\alpha^{a}$ calculations.

As it follows from (1) the uncertainty in computation of aerosol extinction $\varepsilon_{\alpha}$ due to the choice of EAE depends on the ratio $\left(\frac{\lambda_{R}}{\lambda_{L}}\right)^{A}$ and for constant $A$ this uncertainty is range independent. The effect of the EAE on backscattering coefficient is described by the exponent term in (2), which for height independent $A$ can be written as

$\exp \left\{\int_{z_{0}}^{z} \alpha_{L}\left(z^{\prime}\right)\left[1-\left(\frac{\lambda_{L}}{\lambda_{R}}\right)^{A}\right] d z^{\prime}\right\}=\exp \left\{D(z)\left[1-\left(\frac{\lambda_{L}}{\lambda_{R}}\right)^{A}\right]\right\}$

Where $D(z)=\int_{z_{0}}^{z} \alpha_{L}\left(z^{\prime}\right) d z^{\prime}$ is the aerosol optical depth for height interval $\left[\mathrm{z}_{0}, \mathrm{z}\right]$. Thus the uncertainties $\varepsilon_{\beta}$ of backscattering coefficient calculation are integrated over distance $z_{0^{-}} z$ and are the most significant at low heights. If $\Delta A$ is deviation of EAE from "true" value, $\varepsilon_{\beta}$ can be calculated as

$$
\begin{aligned}
& \varepsilon_{\beta}=\frac{\beta^{a}(A+\Delta A)-\beta^{a}(A)}{\beta^{a}(A)}=\frac{\beta^{T}(A+\Delta A)-\beta^{T}(A)}{\beta^{T}(A)-\beta^{m}}= \\
& =\frac{R}{R-1}\left(\exp \left\{D\left(\frac{\lambda_{L}}{\lambda_{R}}\right)^{A}\left(1-\left(\frac{\lambda_{L}}{\lambda_{R}}\right)^{\Delta A}\right)\right\}-1\right)
\end{aligned}
$$

Here $\beta^{T}=\beta^{a}+\beta^{m}$ and $R$ is the scattering ratio $\frac{\beta^{T}}{\beta^{m}}$. For high aerosol loading, when $\beta_{L}^{a} \gg \beta_{L}^{m}$ the ratio $\frac{R}{R-1} \approx 1$ and $\varepsilon_{\beta}$ rises quickly with increasing optical depth $\mathrm{D}$ and $\triangle A$. However in the UV and visible spectral ranges the magnitude of molecular and aerosol backscattering outside of clouds can be comparable, so the influence of molecular scattering on $\varepsilon_{\beta}$ must be considered.

For estimating the uncertainty $\varepsilon_{\beta}$ for different laser wavelengths and aerosol loadings, numerical simulation was performed. The particle bimodal size distribution was represented as:

$$
\frac{d n(r)}{d \ln (r)}=\sum_{i=f, c} \frac{N_{i}}{(2 \pi)^{1 / 2} \cdot \ln \sigma_{i}} \cdot \exp \left[\frac{\left(\ln r-\ln r_{0 i}\right)^{2}}{2\left(\ln \sigma_{i}\right)^{2}}\right]
$$

Here $N_{i}$ is the total particle number of the $i$-th mode, $r_{i}$ describes the mode radius and $\ln \sigma_{i}$ is the dispersion. The index $i=f, c$ corresponds to the fine and coarse mode, respectively. For all computations the values $r_{0 f}=0.1 \mu \mathrm{m}, r_{0 c}=1.2 \mu \mathrm{m}$ and $\ln \sigma_{f, c}=0.4$ were used and the complex refractive index (CRI) was assumed to be the same for both modes. The concentration of particles in the find mode $\mathrm{N}_{\mathrm{f}}$ was kept constant through all simulations, while the concentration in the coarse mode varied. The spectral dependence of the EAE is influenced by the relative contributions of the fine and the coarse modes, so to evaluate this effect three types of particle size distribution (PSD) were considered. For the first type (T1) with $\frac{N_{f}}{N_{c}}=10^{4}$ the main particle volume is attributed to the fine mode. For the second type (T2) $\frac{N_{f}}{N_{c}}=10^{3}$ the total volume of particles in each mode is comparable. And finally, in the distribution T3 with $\frac{N_{f}}{N_{c}}=10^{2}$ the coarse mode is predominant.

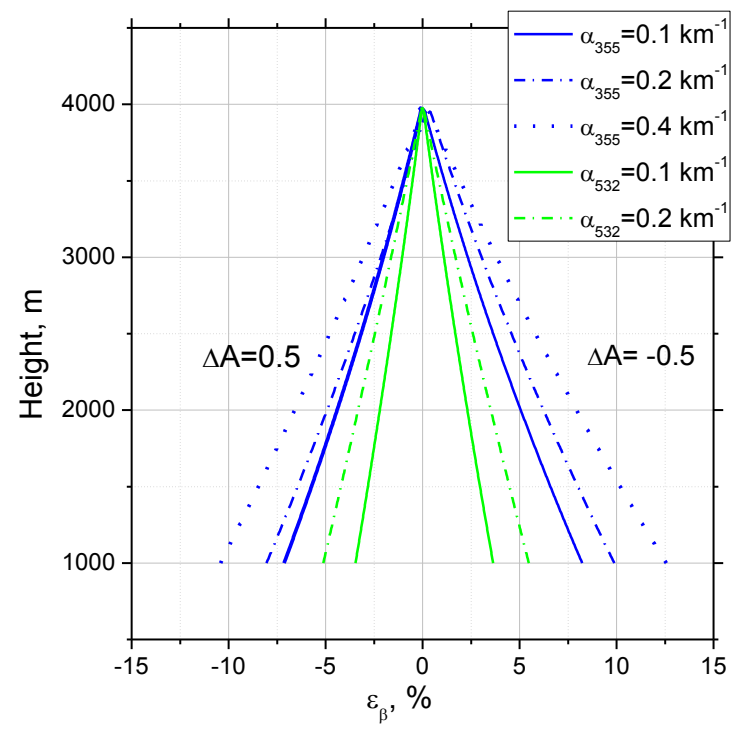

Fig.1. Profiles of backscattering coefficient uncertainties at $355 \mathrm{~nm}$ (blue) and $532 \mathrm{~nm}$ (green) for extinction coefficient $0.1 \mathrm{~km}^{-1}$ (solid), $0.2 \mathrm{~km}^{-1}$ (dash-dot), $0.4 \mathrm{~km}^{-1}$ (dot). Results are given for $\Delta \mathrm{A}= \pm 0.5$.

A Raman lidar based on a tripled Nd:YAG laser can measure the EAE at $355-532 \mathrm{~nm}$ wavelengths $\left(A_{355}\right.$ ${ }_{532}$ ). However, when nitrogen vibrational Raman backscatters at $387 \mathrm{~nm}$ and $608 \mathrm{~nm}$ are used to get backscattering coefficients at $355 \mathrm{~nm}$ and $532 \mathrm{~nm}$, the Ångström exponents $A_{355-387}$ and $A_{532-608}$ are needed and these may differ from $A_{355-532}$. Table 1 shows the Ångström exponents $A_{355-532}, A_{355-387}$, $A_{532-608}$ for the size distributions T1-T3 and refractive indices $\mathrm{m}=1.35$-i0.005; 1.45 -i0.005; 1.6 -i0.005; 1.6 i0.05. These values of CRI correspond to variety of 
particles, such as water aerosol, biomass burning products and desert dust (Dubovik et al., 2002). The spectral dependence of the EAE is the most significant for particles with a fine mode predominance. For the $\mathrm{T} 1$ distribution, the uncertainty in the choice of the Ångström exponent $\Delta A$ may exceed 0.5 when $\mathrm{A}_{355-532}$ is used for the computation of both $\beta_{355}$ and $\beta_{532}$. For particles with a coarse mode predominance (T3), the spectral dependence of the Ångström exponent is weaker and $\Delta A<0.2$ when $\mathrm{A}_{355-532}$ is used.

Table 1. Extinction Ångström exponents for different size distributions and different refractive indices.

\begin{tabular}{|l|c|c|c|c|}
\hline Aerosol & \multirow{2}{*}{$\begin{array}{c}\text { Refractive } \\
\text { index }\end{array}$} & \multicolumn{3}{|c|}{ Angström exponent } \\
\cline { 2 - 5 } & $\mathrm{A}_{355-532}$ & $\mathrm{~A}_{355-387}$ & $\mathrm{~A}_{532-608}$ \\
\hline \multirow{4}{*}{$\mathrm{T} 1$} & $1.35-\mathrm{i} 0.005$ & 1.62 & 1.39 & 1.98 \\
\cline { 2 - 5 } & $1.45-\mathrm{i} 0.005$ & 1.32 & 1.03 & 1.79 \\
\cline { 2 - 5 } & $1.6-\mathrm{i} 0.005$ & 0.87 & 0.52 & 1.47 \\
\cline { 2 - 5 } & $1.6-\mathrm{i} 0.05$ & 0.78 & 0.46 & 1.30 \\
\hline \multirow{4}{*}{$\mathrm{T} 2$} & $1.35-\mathrm{i} 0.005$ & 1.40 & 1.24 & 1.58 \\
\cline { 2 - 5 } & $1.45-\mathrm{i} 0.005$ & 1.15 & 0.92 & 1.46 \\
\cline { 2 - 5 } & $1.6-\mathrm{i} 0.005$ & 0.81 & 0.48 & 1.33 \\
\cline { 2 - 5 } & $1.6-\mathrm{i} 0.05$ & 0.72 & 0.43 & 1.18 \\
\hline \multirow{4}{*}{$\mathrm{T} 3$} & $1.35-\mathrm{i} 0.005$ & 0.58 & 0.59 & 0.48 \\
\cline { 2 - 5 } & $1.45-\mathrm{i} 0.005$ & 0.426 & 0.41 & 0.59 \\
\cline { 2 - 5 } & $1.6-\mathrm{i} 0.005$ & 0.44 & 0.28 & 0.64 \\
\cline { 2 - 5 } & $1.6-\mathrm{i} 0.05$ & 0.39 & 0.24 & 0.57 \\
\hline
\end{tabular}

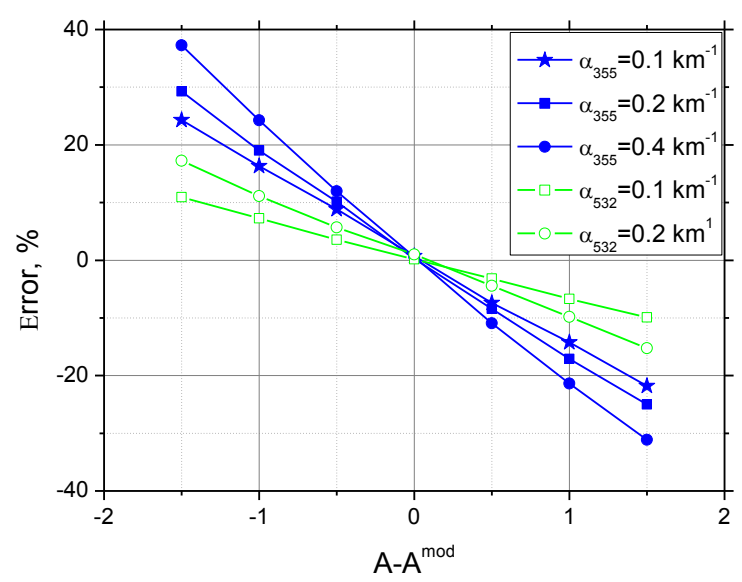

Fig.2. The errors of backscattering coefficient calculation at $355 \mathrm{~nm}$ (blue) and $532 \mathrm{~nm}$ (green) as a function of deviation from model value of Angstrom exponent.

To estimate how sensitive the backscattering coefficient is to the choice of the Ångström exponent, we considered an aerosol layer extending from the ground up to $4000 \mathrm{~m}$ with a height independent particle concentration. As mentioned, the spectral dependence of $A$ has the most influence for PSD with a predominant fine mode, so the simulation results are presented for the $\mathrm{T} 1$ distribution. The uncertainty of the backscattering calculation depends on aerosol loading, so in the simulations different particle concentrations were considered in order to obtain extinction coefficients of $\alpha^{\mathrm{a}}=0.1 \mathrm{~km}^{-1}, 0.2 \mathrm{~km}^{-1}, 0.4$ $\mathrm{km}^{-1}$ at $355 \mathrm{~nm}$ and $532 \mathrm{~nm}$. The refractive index used was $\mathrm{m}=1.45$-i.005, and corresponding model values of $A_{355-387}, A_{532-608}$ and $A_{355-532}$ were taken from Table 1. The backscattering and extinction coefficients for the chosen size distribution were used to generate noise-free synthetic lidar signals. Then, the values of $\alpha$ and $\beta$ were calculated using Ångström exponents $A$ which differed from the model values $A^{\text {mod }}$. The systematic uncertainty for backscattering was determined as $\varepsilon_{\beta}=\frac{\beta^{a}(A)-\beta^{a}\left(A^{\mathrm{mod}}\right)}{\beta^{a}\left(A^{\mathrm{mod}}\right)}$. The systematic uncertainty in extinction was defined similarly.

Table 2. Uncertainties of extinction $\varepsilon_{\alpha}$ at 355 and $532 \mathrm{~nm}$ for different values of Angström exponent $A$ chosen in computations. Results are shown for $\mathrm{m}=1.45-\mathrm{i} 0.005$.

\begin{tabular}{|c|c|c|c|c|}
\hline \multirow{2}{*}{$A$} & \multicolumn{2}{|c|}{$355 \mathrm{~nm}$} & \multicolumn{2}{c|}{$532 \mathrm{~nm}$} \\
\cline { 2 - 5 } & $A-A^{\text {mod }}$ & $\varepsilon_{\alpha}$ & $A-A^{\text {mod }}$ & $\varepsilon_{\alpha}$ \\
\hline 0 & -1 & $4.5 \%$ & -1.8 & $10.5 \%$ \\
\hline 0.5 & -0.5 & $2 \%$ & -1.3 & $7.5 \%$ \\
\hline 1 & 0 & $0.15 \%$ & -0.8 & $4.5 \%$ \\
\hline 1.5 & 0.5 & $2 \%$ & -0.3 & $1.5 \%$ \\
\hline 2 & 1 & $4 \%$ & 0.2 & $1.2 \%$ \\
\hline
\end{tabular}

The results of the simulations for particle extinction are summarized in Table 2, showing $\varepsilon_{\alpha}$ at 355 and $532 \mathrm{~nm}$ for different assumed values of $A$ in range [0, 2.0]. As mentioned, the uncertainty of extinction $\varepsilon_{\alpha}$ is independent of range or aerosol loading and in our computations it doesn't exceed $2 \%$ for both wavelengths, when $A_{355-532}$ is used.

In contrast to extinction the uncertainties $\varepsilon_{\beta}$ depend on range since the differential transmission between the two Raman wavelengths is involved and errors in this calculation accumulate with range. The height profiles of $\varepsilon_{\beta}$ at $355 \mathrm{~nm}$ for the particle extinction coefficients $0.1 \mathrm{~km}^{-1}, 0.2 \mathrm{~km}^{-1}$ and $0.4 \mathrm{~km}^{-1}$ are given in Fig.1 for $\Delta A=A-A^{\bmod }= \pm 0.5$. For comparison, the same figure also shows results at $532 \mathrm{~nm}$ for $\alpha=0.1 \mathrm{~km}^{-1}$ and $0.2 \mathrm{~km}^{-1}$. The uncertainty is negligible near the reference height $\left(\mathrm{z}_{0}=4000 \mathrm{~m}\right)$ and it rises with increasing $\mathrm{z}-\mathrm{z}_{0}$. At $\mathrm{z}=1000 \mathrm{~m}$ the uncertainties can be as high as $8 \%$ and $10 \%$ at 355 $\mathrm{nm}$ for $\alpha=0.1$ and $0.2 \mathrm{~km}^{-1}$ respectively, when $\Delta \mathrm{A}=$ 0.5 . For $532 \mathrm{~nm}$ these uncertainties are $4 \%$ and $6 \%$, which is significantly lower than at $355 \mathrm{~nm}$. An increase of extinction up to $\alpha=0.4 \mathrm{~km}^{-1}$ leads to a rapid increase in $\varepsilon_{\beta}$ and the correct choice of the 
Ångström exponent becomes more important. Fig.2 illustrates the dependence of $\varepsilon_{\beta}(\Delta A)$ at $\mathrm{z}=1000 \mathrm{~m}$ for different values of extinction coefficient. This dependence is close to linear so an increase of $\Delta A$ to $\Delta A=1$ doubles the uncertainty, when compared with Fig.1.

\section{ANALYSIS OF LIDAR MEASUREMENTS}

The observations performed with multiwavelength GSFC Raman lidar in August 2013 [2] were used to analyze sensitivity of $\alpha$ and $\beta$ calculation to the choice of the Ångström exponent. During the period considered a strong fire smoke event occurred and the aerosol layers extended up to $4000 \mathrm{~m}$ height. The Ångström exponent $\mathrm{A}_{355-532}$ varied with height from 0.5 to 2.0, as shown in Fig.3. $\mathrm{A}_{355-532}$ was computed for values of Ångström exponent in the range 0.5-2.0 assuming AEA to be height independent. The profile of $A_{355-532}$ demonstrates low sensitivity to the choice of EAE, while sensitivity of backscattering to AEA is high, as shown in Fig.4. At $1000 \mathrm{~m}$ height the deviation of $\beta_{355}$ from mean value may achieve $10 \%$ depending on EAE chosen. For extinction the uncertainty is below $5 \%$.

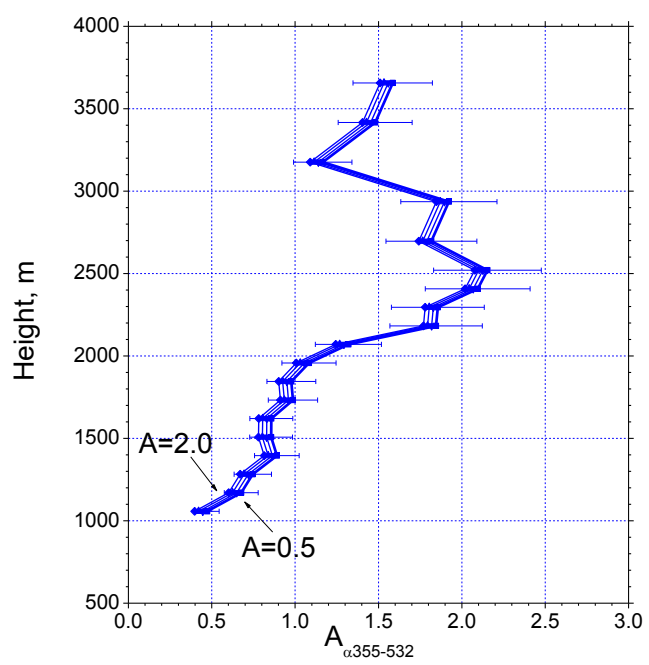

Fig.3. Vertical profile of $A_{355-532}$ computed for values of Ångström exponent in the range 0.5-2.0.

\section{CONCLUSION}

The simulations performed here demonstrate that the ambiguity in the choice of the Ångström exponent can be a source of significant uncertainties in backscattering calculation when the aerosol layers with high optical depth are profiled and that this effect is especially pronounced at $355 \mathrm{~nm}$. In real measurements, the situation can be even more complicated because of the vertical variability of the Ångström exponent. These uncertainties can be eliminated through the use of rotational Raman scattering instead of vibrational one, thus decreasing the frequency shift of the inelastically scattered component. The first results of rotational Raman channel operation will be reported in accompanying presentation.
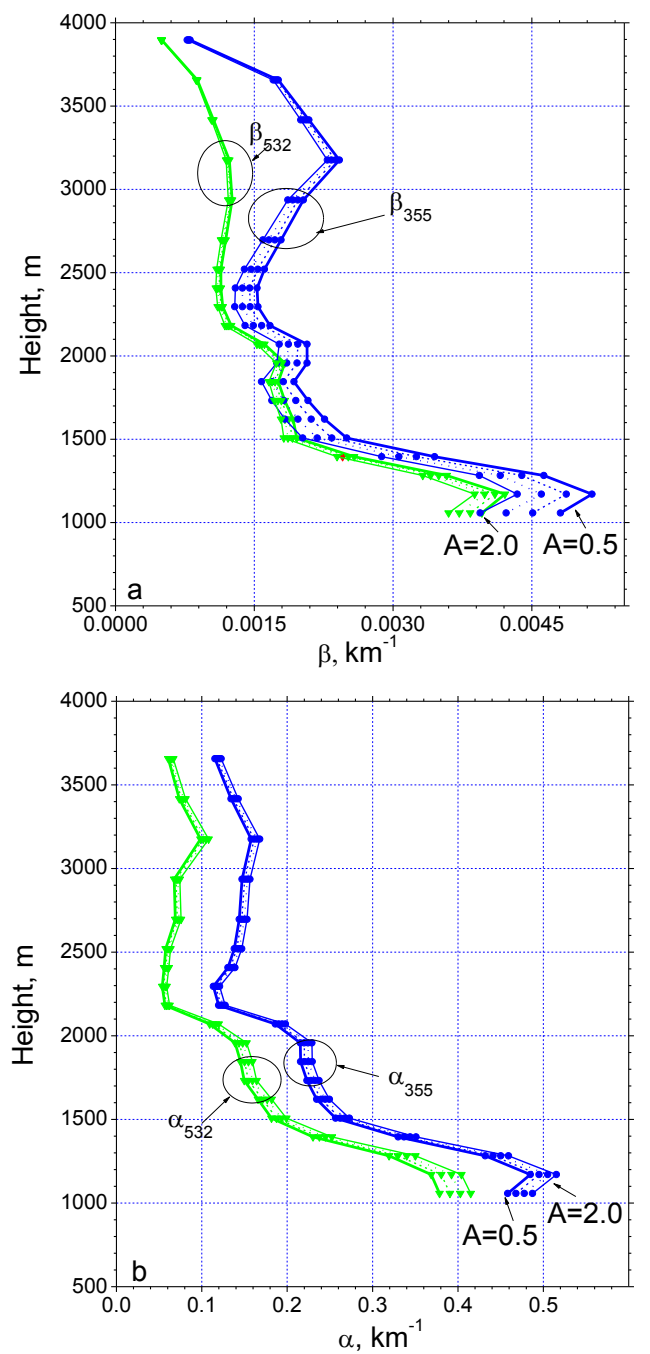

Fig.4. Vertical profiles of (a) backscattering and (b) extinction coefficient computed for values of Ångström exponent in the range 0.5-2.0.

\section{REFERENCES}

[1] Ansmann, et al.: Combined Raman elasticbackscatter lidar for vertical profiling of moisture, aerosols extinction, backscatter, and lidar ratio, Appl.Phys.B, 55, 18-28, 1992.

[2] I. Veselovskii, et al. "Characterization of forest fire smoke event near Washington, D.C. in Summer 2013 with multi-wavelength lidar", Atmos. Chem. Phys. 15, 1647-1660, 2015. 